\title{
Ischiofemoral Impingement Syndrome
}

\author{
Soyoung Lee, $\mathrm{MD}^{1}$, Inhwan Kim, $\mathrm{MD}^{1}$, Sung Moon Lee, $\mathrm{MD}^{2}$, Jieun Lee, $\mathrm{MD}^{1}$
}

Departments of ${ }^{1}$ Rehabilitation Medicine and ${ }^{2}$ Radiology, Keimyung University School of Medicine, Daegu, Korea

Ischiofemoral impingement syndrome is known as one of the causes of hip pain due to impingement of ischium and femur, and usually correlated with trauma or operation. We report a rare case of ischiofemoral impingement syndrome that has no history of trauma or surgery. A 48-year-old female patient was referred for 2 months history of the left hip pain, radiating to lower extremity with a hip snapping sensation. She had no history of trauma or surgery at or around the hip joint and femur. The magnetic resonance imaging (MRI) of the lumbar spine showed no abnormality, except diffuse bulging disc without cord compression at the lumbosacral area. Electrophysiologic study was normal, and there were no neurologic abnormalities compatible with the lumbosacral radiculopathy or spinal stenosis. Hip MRI revealed quadratus femoris muscle edema with concurrent narrowing of the ischiofemoral space. The distance of ischiofemoral space and quadratus femoris space were narrow. It was compatible with ischiofemoral impingement syndrome. After treatment with nonsteroidal anti-inflammatory drugs, physical therapy, and exercise program, the patient's pain was relieved and the snapping was improved. To our knowledge, this is the first reported case of a nontraumatic, noniatrogenic ischiofemoral impingement syndrome, and also the first case to be treated by a nonsurgical method in the Republic of Korea.

Keywords Ischiofemoral, Impingement, Impingement syndrome

\section{INTRODUCTION}

There are several causes of hip pain, with the impingement of ischium and femur being one. The impingement between these structures is rare, considering that the distance between the lesser trochanter and ischium is approximately $20 \mathrm{~mm}[1]$. The condition is usually correlated with trauma or prior hip surgery, and the patients

Received December 27, 2011; Accepted June 8, 2012

Corresponding author: Jieun Lee

Department of Rehabilitation Medicine, Dongsan Medical Center, Keimyung University School of Medicine, 56 Dalseong-ro, Jung-gu, Daegu 700-712, Korea

Tel: +82-53-250-7264, Fax: +82-53-250-7268, E-mail: withjieunlee@ hanmail.net

() This is an open-access article distributed under the terms of the Creative Commons Attribution Non-Commercial License (http://creativecommons. org/licenses/by-nc/3.0) which permits unrestricted noncommercial use, distribution, and reproduction in any medium, provided the original work is properly cited.

Copyright $\odot 2013$ by Korean Academy of Rehabilitation Medicine obtain pain relief with a lesser trochanter excision [2,3]. By using magnetic resonance imaging (MRI) of the hip, we can detect a decline of distance between the ischial tuberosity and lesser trochanter, and the abnormal signal intensity of the quadratus femoris muscle [4].

We report a rare case of the ischiofemoral impingement syndrome that has no history of trauma or surgery, and has been treated with a nonsurgical method, including the nonsteroidal anti-inflammatory drugs, physical therapy, and exercise program.

\section{CASE REPORT}

A 48-year-old female patient visited the hospital due to a left hip pain, radiating to the lower extremity with a hip snapping sensation for 2 months, especially on walking. There was no symptom on the right side. She was able to walk independently and bear weight on the affected 
side, but complained of more pain on the weight bearing status. She had no history of lower extremity trauma or surgery except hypertension due to primary aldosteronoma. In physical examination, a range of motion of the hip internal and external rotation was decreased slightly without pain. She had strong palpable deep peroneal artery and audible digital artery flow bilaterally. There was no motor weakness, but there was a sensory change on the lateral aspect of the leg and dorsum of foot, compatible with the L5 dermatomal area. She had normal deep tendon reflex bilaterally on knee jerk and ankle jerk. Babinski sign and ankle clonus were absent. Straight leg raising test was decreased to 30 degrees only on the left side. Lasegue test and Patrick sign were positive on the left side, but negative on the right side.

We requested radiographs and MRI of the lumbar spine for further evaluation. MRI of the spine showed no ab-

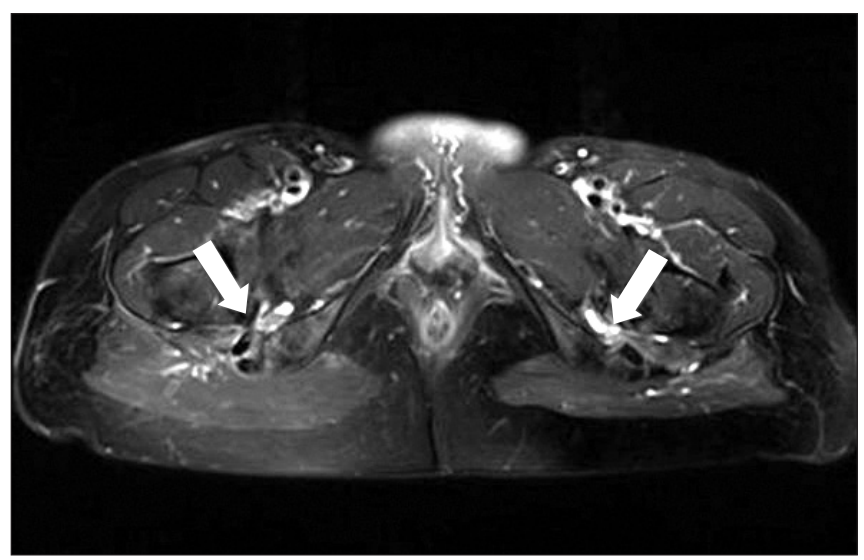

Fig. 1. Axial T2-weighted fat-suppressed magnetic resonance image of both hips shows diffuse edema and increased signal intensity within the quadratus femoris muscle bilaterally (arrows). normality except diffuse and mild bulging disc without cord compression at the lumbosacral area. The radiographs of pelvis in anteroposterior and frog lateral projection showed no abnormal finding. Electrophysiologic study showed normal findings. There were no abnormal results compatible to the lumbosacral radiculopathy or spinal stenosis. At last, MRI of the hip was performed. Hip MRI revealed bilaterally increased signal intensity of the quadratus femoris muscle with concurrent narrowing of the ischiofemoral space. On axial T2-weighted fatsuppressed magnetic resonance images (Fig. 1), there were diffuse edema and increased signal intensity within the quadratus femoris muscle bilaterally. The distance of ischiofemoral space was $12.96 \mathrm{~mm}$ on the right side and $10.24 \mathrm{~mm}$ on the left side. The distance of quadratus femoris space was $6.17 \mathrm{~mm}$ on the right side and $4.31 \mathrm{~mm}$ on the left side (Fig. 2). There were no abnormal findings, such as degenerative arthritis, femoroacetabular impingement, or osteonecrosis, as possible causes of other hip pain origins. It was compatible with ischiofemoral impingement syndrome bilaterally. She complained the hip pain only on the left side, not the right side.

After being diagnosed with the ischiofemoral impingement syndrome, the patient was hospitalized for a treatment over four weeks. Nonsteroidal anti-inflammatory drugs and gabapentin were prescribed for pain relief. Hot pack, ultrasound, and interferential current therapies were applied around the hip area. She received an exercise program for stretching of the hip muscle and connective tissues. The exercise program was targeted to the quadriceps femoris, piriformis, and other hip muscles, in order to adequately reduce pain and increase the range of motion in the hip joint. After discharge, the patient maintained the drug regimen and exercise program. The
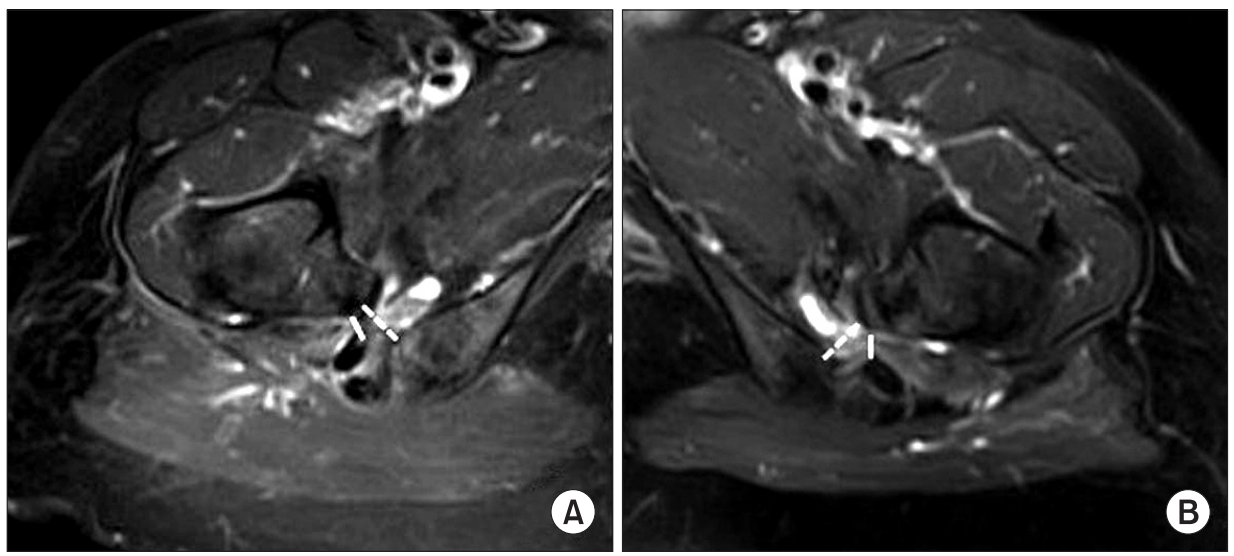

Fig. 2. The ischiofemoral space on axial magnetic resonance (MR) images is $12.96 \mathrm{~mm}$ in the right side and $10.24 \mathrm{~mm}$ in the left side (dotted line). The quadratus femoris space on axial MR images is $6.17 \mathrm{~mm}$ in the right side and 4.31 $\mathrm{mm}$ in the left side (solid line). (A) is the right side and (B) is the left side. 
initial visual analogue scale (VAS) was 7 to 8 . After six weeks of treatment, pain of the left hip was decreased gradually to VAS 2 to 3 . The range of motion in the hip joint was normalized, and the hip snapping sensation was resolved.

\section{DISCUSSION}

Generally, the ischiofemoral impingement syndrome rarely occurs, but as a potential cause of hip pain after a hip surgery or trauma $[2,3]$. In the previous studies, there have been noted abnormalities after the total hip arthroplasty, proximal femoral osteotomy, and abduction injury to the hip $[2,3]$. Patients of previous reports were treated with resection of the lesser trochanter, with a complete pain relief $[2,3]$. In a recent study, only one case of ischiofemoral impingement syndrome with no history of the lower extremity trauma or surgery was reported [1].

The etiology of ischiofemoral impingement is uncertain, but there are acquired and congenital causes. Several acquired causes include intertrochanteric fractures with involvement of the lesser trochanter, valgus producing intertrochanteric osteotomy, and osteoarthritis leading to superior and medial migration of the femur [2]. Without the mention of any acquired bony abnormalities, the cause of ischiofemoral narrowing can be congenital or positional [3].

It has been reported that the narrowing of ischiofemoral space and quadratus femoris space in MRI of the hip can be diagnosed as ischiofemoral impingement syndrome [4]. The lesser trochanter and ischial tuberosity are about $20 \mathrm{~mm}$ apart with the hip in adduction, external rotation, and extension [2]. This is why the femur can rotate without any influence of the ischial tuberosity or proximal hamstring tendon [4]. Conversely, the narrowing of the ischiofemoral space leads to edema or tear of the quadratus femoris muscle and other hip muscles, especially the hamstring muscle. The most sensitive and specific measurement of the narrowed ischiofemoral space is below $17 \mathrm{~mm}$, and measurement of the quadratus femoris space is below $8 \mathrm{~mm}$ [4]. In our case, the ischiofemoral space is $12.96 \mathrm{~mm}$ in the right side and $10.24 \mathrm{~mm}$ in the left side. Quadratus femoris space is $6.17 \mathrm{~mm}$ in the right side and $4.31 \mathrm{~mm}$ in the left side. The abnormal relationship between the lesser trochanter and ischium may lead to injury intervening soft tissues, such as quadratus femoris muscle [1], and the narrowing of the ischiofemoral space may attribute to edema of the quadratus femoris muscle [5]. This situation leads to the potential hip pain and referred pain. The referred pain can be developed by irritation of sciatic nerve caused by the proximity of the edematous quadratus femoris [1]. In our case, MRI of the hip showed increased signal intensity of quadratus femoris muscle bilaterally and the abnormal narrowing of the ischiofemoral and quadratus femoris space bilaterally, but the distance was shorter on the left. The patient had the left hip pain radiating to the lower extremity with a hip snapping sensation, and had no history of trauma or surgery. The range of the motion of the hip was mildly decreased bilaterally without any acquired bony abnormality.

Although the patient complained of the left hip pain, we needed to apply not only the therapeutic but also preventive treatment because as the right hip could provoke pain in the near future. We prescribed nonsteroidal anti-inflammatory drugs, gabapentin for radiating pain, physical modalities, and exercise programs for stretching of the hip muscles.

In the hip pain with radiating leg pain, we need to consider lumbosacral radiculopathy, piriformis syndrome and other causative factors such as spinal stenosis and ischiofemoral impingement syndrome [6-8]. The diagnosis of ischiofemoral impingement syndrome need to be considered in patients complaining of the hip pain with a snapping hip. Delayed diagnosis of ischiofemoral impingement syndrome can lead to a chronic process of hip muscles and other structures around the hip joint [4]. It can be bursa-like formation surrounding the lesser trochanter or fatty infiltration in the quadratus femoris [4]. This is the first report in Korea on the ischiofemoral impingement syndrome without any acquired bony abnormalities, and managed with a nonsurgical approach.

The diagnosis of the ischiofemoral impingement is complex. It is important to have a focus on the hip pain with a radiating pain, through the history taking, physical examination, and appropriating studies of the hip. If it is early diagnosed before any structural or pathologic changes of the hip muscles and surrounding structures, the ischiofemoral impingement syndrome may be treated with nonsurgical method.

\section{CONFLICT OF INTEREST}

No potential conflict of interest relevant to this article 
was reported.

\section{REFERENCES}

1. Patti JW, Ouellette H, Bredella MA, Torriani M. Impingement of lesser trochanter on ischium as a potential cause for hip pain. Skeletal Radiol 2008;37:939-41.

2. Johnson KA. Impingement of the lesser trochanter on the ischial ramus after total hip arthroplasty: report of three cases. J Bone Joint Surg Am 1977;59:268-9.

3. Ali AM, Whitwell D, Ostlere SJ. Case report: imaging and surgical treatment of a snapping hip due to ischiofemoral impingement. Skeletal Radiol 2011;40:653-6.

4. Torriani M, Souto SC, Thomas BJ, Ouellette H, Bredel- la MA. Ischiofemoral impingement syndrome: an entity with hip pain and abnormalities of the quadratus femoris muscle. AJR Am J Roentgenol 2009;193:18690.

5. Kassarjian A. Signal abnormalities in the quadratus femoris muscle: tear or impingement? AJR Am J Roentgenol 2008;190:W379.

6. Milette PC, Fontaine S, Lepanto L, Breton G. Radiating pain to the lower extremities caused by lumbar disk rupture without spinal nerve root involvement. AJNR Am J Neuroradiol 1995;16:1605-13.

7. Kirschner JS, Foye PM, Cole JL. Piriformis syndrome, diagnosis and treatment. Muscle Nerve 2009;40:10-8.

8. Genevay S, Atlas SJ. Lumbar spinal stenosis. Best Pract Res Clin Rheumatol 2010;24:253-65. 